\title{
Clinical course of grafted cartilage in osteoarticular frozen autografts for reconstruction after resection of malignant bone tumor involving an epiphysis
}

Katsuhiro Hayashi ( $\sim$ khayashi830@gmail.com )

Department of Orthopaedic Surgery, Graduate School of Medical Sciences, Kanazawa University https://orcid.org/0000-0001-8665-2154

Norio Yamamoto

Kanazawa Daigaku

Akihiko Takeuchi

Kanazawa Daigaku

Shinji Miwa

Kanazawa Daigaku

Kentaro Igarashi

Kanazawa Daigaku

Takashi Higuchi

Kanazawa Daigaku

Kensaku Abe

Kanazawa Daigaku

Yuta Taniguchi

Kanazawa Daigaku

Hisaki Aiba

Kanazawa Daigaku

Yoshihiro Araki

Kanazawa Daigaku

Hirotaka Yonezawa

Kanazawa Daigaku

Sei Morinaga

Kanazawa Daigaku

Hiroyuki Tsuchiya

Kanazawa Daigaku

Research article

Keywords: Epiphyseal reconstruction, sarcoma, frozen autograft, recycled bone, cartilage 
Posted Date: October 4th, 2019

DOI: https://doi.org/10.21203/rs.2.15618/v1

License: (c) (i) This work is licensed under a Creative Commons Attribution 4.0 International License. Read Full License 


\section{Abstract}

Background: There are several options for biological reconstruction after bone tumor resection. If the tumor invades an epiphysis, the reconstruction is far more complicated because there is no option to restore large joint cartilage using currently available medical techniques. Frozen autograft with liquid nitrogen has been used as recycled autologous bone graft and the purpose of the present study was to assess the outcome of grafted cartilage in osteoarticular frozen autografts used in the treatment of patients with bone and soft-tissue sarcoma. Method: We have treated 27 patients with cases of bone tumor resection involving an epiphysis where frozen autografts were used for reconstruction. If the tumor was located in a limited part of the epiphysis, partial resection of the epiphysis was performed to preserve the healthy part of the cartilage in 4 cases. The survival of grafted frozen cartilage was assessed by X-ray imaging. The end point was defined as grade IV of the Kellgren and Lawrence osteoarthritis grading system and was recorded using the Kaplan-Meier method. In case of removal of grafted bone after the surgery, pathological findings were assessed by hematoxylin and eosin staining of sections of resected cartilage in available cases to observe the fate of grafted cartilage over time. Result: The postoperative mean follow-up period was 94.0 months. Grade IV osteoarthritis occurred in 12 patients. All patients in the partial epiphyseal freezing group survived compared with only 1 patient in the total epiphyseal freezing group who had survived to the final follow up $(p<0.01)$. Resected specimens with grafted cartilage were examined histologically. A sample excised after 14 months had dead cartilage with empty lacunae and the surface of the cartilage had reactive fibrous tissue. Conclusion: Grafted cartilage of frozen osteoarticular autografts was worn out over time. However, intraepiphyseal resection preserving partial healthy cartilage resulted in excellent survival. This technique requires careful planning of the surgery but might be an alternative to megaprosthesis.

\section{Background}

There are several options for reconstruction after bone tumor resection, including an endoprosthesis, allogenic bone graft, recycling autologous bone graft, vascularized bone graft, and distraction osteogenesis. The ideal reconstruction should have life-long durability, non-limited activity, resistance to infection, reasonable cost, and relatively undemanding surgery. When the tumor is located in a diaphysis, reconstruction is relatively simple because only bone reconstruction is required. However, if the tumor invades an epiphysis, the reconstruction is far more complicated because there is no option to restore large joint cartilage using currently available medical techniques. Use of an endoprosthesis is a common procedure for joint reconstruction, but it is not an ultimate goal because a prosthesis is not a permanent, durable reconstruction and its function is not perfectly comparable to that of a normal limb [1]. Biological reconstruction could offer a superior clinical outcome; for instance, distraction osteogenesis can regenerate normal bone structure [2], but it can regenerate only bone and not cartilage. In some institutions, osteoarticular allograft or recycled osteoarticular autograft is used for epiphysial reconstruction instead of an endoprosthesis. Because the grafted cartilage is dead in such cases, there is great interest in the fate of the grafted cartilage. Jamshidi reported that the 10-year survival rate of an 
osteoarticular allograft in pediatric patients was $62.2 \%$ [3]. Ultimately, the cartilage will be lost after grafting.

Frozen autograft with liquid nitrogen has been used as recycled autologous bone graft for reconstruction after bone tumor surgery [4]. The development of this method was based on studies in vitro and in vivo of the hypothermic effects of liquid nitrogen on the proliferation of osteosarcoma cells. Freezing devitalises tumor cells by inducing ice crystal formation and cell dehydration. There are several advantages of frozen autografts such as the short time to bony union after using frozen tissue autografting compared with that for autoclaved autografts [5]. Takata reported that the activity of bone morphogenetic proteins was preserved in frozen autografts treated with liquid nitrogen compared with those treated with heat [6]. There are also reports that antitumor immunity could be enhanced by cryotreatment [7] .

A surgical technique that involves partial excision of the bone-containing tumor, followed by rotation of the limb to facilitate its immersion in liquid nitrogen, to create a frozen autograft has been developed. Nitrogen in this form is readily available and with this technique early post-operative functional recovery using fewer osteotomy sites, and a lower rate of complication related to the graft have been achieved [8].

The clinical outcome of frozen autografts has been reported, and frozen autografts achieved a composite graft leading to a stable result compared with an osteoarticular graft for reconstruction of an epiphysis $[9,10]$. However, there are still good cases of osteoarticular grafts where the grafted cartilage survived for a relatively long period with good function. The surgical techniques for tumor surgery are still being developed; for instance, hemi-cortical excision is applied in selected cases because of the assessment of efficacy of preoperative chemotherapy for malignant bone tumor has been established $[11,12]$. To our knowledge, there are no reports of the fate of grafted non-vital cartilage in recycling autografts. We had several cases in which we were able to evaluate the grafted cartilage by histology after resection.

The purpose of the present study was to assess the outcome of grafted cartilage in osteoarticular autografts treated by liquid nitrogen used in the treatment of patients with bone and soft-tissue tumor.

\section{Methods}

\section{Patients}

We have treated 71 patients with cases of bone tumor resection involving an epiphysis where frozen autografts were used for reconstruction. The operations were performed between 1999 and 2016 (Table 1). To replace the cartilage, 27 cases were reconstructed as osteoarticular autografts after freezing tumorbearing bone and 44 cases were reconstructed using a composite graft of frozen bone and a prosthesis. The first case of reconstruction with a composite graft was performed in 2003 and the procedure gradually replaced that for osteoarticular grafts (Supplementary Fig).

In the present study, 27 cases in which grafted frozen cartilage was used were examined including those in 19 men and 8 women, with a mean age of 31.6 years (range 12 to 72 years). The diagnoses were 17 
osteosarcomas, 3 chondrosarcomas, 3 metastatic tumors, 2 Ewing's sarcomas, and 2 undifferentiated pleomorphic sarcomas. Tumours were located in the pelvis including acetabulum in 10, distal femur in 7 , proximal tibia in 6, proximal humerus in 2, proximal femur in 1, and knee joint in 1.

\section{Surgery}

The surgical procedure has been described in detail previously [5]. In brief, after the excision of the bone tumor, the tumor-bearing bone was frozen in liquid nitrogen for $20 \mathrm{~min}$, then thawed at room temperature in distilled water for $15 \mathrm{~min}$. The frozen autograft including cartilage was used for the reconstruction. If the tumor was located in a limited part of the epiphysis, partial resection of the epiphysis was performed to preserve the healthy part of the cartilage in 4 cases.

Immediately after the surgery, patients were allowed range of movement exercise. Partial weight-bearing was allowed 2 months after surgery, and weight protection was continued until sufficient callus formation at the host-graft junction occurred.

\section{Statistical analysis}

The survival of grafted frozen cartilage was assessed by X-ray imaging and the end point is defined as grade IV of the Kellgren and Lawrence osteoarthritis grading system. Survival of grafted cartilage was recorded using the Kaplan-Meier method and a log-rank test was used to determine significant differences between the group with partial epiphyseal freezing and the group with total epiphyseal freezing. All statistical analyses were performed with EZR software (Saitama Medical Center, Jichi Medical University, Saitama, Japan), which is a graphical user interface for R (The R Foundation for Statistical Computing, Vienna, Austria) [13]. Patients who had died, who had deep infection, or who were lost to follow-up were censored for survival.

\section{Histology}

In case of removal of grafted bone after the surgery, pathological findings were assessed by hematoxylin and eosin staining of sections of resected cartilage in available cases to observe the fate of grafted cartilage over time.

The study received ethical approval from the institutional review boards of our universities.

\section{Results}


The postoperative mean follow-up period was 94.0 months (range 6 to 217 months). Grade IV osteoarthritis occurred in 12 patients, 5 patients maintained, 5 patients became infected, 3 patients died of disease, and 1 patient was lost to follow-up. We resected 2 cases of acetabular tumors throughout the cartilage using a navigation system and in 2 cases of tumors in a distal femur we resected only a medial or lateral condyle preserving the other condyle. All patients in the partial epiphyseal freezing group survived compared with only 1 patient in the total epiphyseal freezing group who had survived to the final follow up $(p=0.00663$, Fig 1$)$.

Resected specimens with grafted cartilage were examined histologically. A sample excised after 6 months showed dead cartilage with empty lacunae and the cartilage had surface fibrillation. A sample excised after 14 months had dead cartilage with empty lacunae and the surface of the cartilage had reactive fibrous tissue (Figs 2,3). Representative cases of cartilage survival or lost are shown in Figs 4-6.

\section{Discussion}

Grafted cartilage of frozen tumor-bearing bone was worn out over time. Frozen autografts and prosthetic composite grafts are considered as more durable reconstruction, however, hemicondylar resection or inter-cartilaginous resection preserving partial healthy cartilage resulted in excellent survival in this study. The technique is demanding and requires careful planning of the surgery. For example, hemicondylar resection and reconstruction require careful indication considering the anatomical arrangement of the weight axis. The reconstruction should recover original anatomy even though the osteotomy usually causes the loss of a few $\mathrm{mm}$ of bone that tends to produce small location differences for the replaced recycled bone. Pelvic osteotomy requires detailed anatomical examinations, otherwise the surgical margin easily becomes positive. In the partial epiphyseal freezing group, we achieved a negative tumor margin, no recurrence, and good survival of the grafted cartilage.

Histology of grafted cartilage showed dead cartilage at 6 and 14 months after frozen autografts. There was some additional fibrous tissue on the surface of the cartilage at 14 months in at least one patient that may have protected the dead cartilage, at least in part. Nevertheless, it cannot be expected that the cartilage will be regenerated. Preventing cryoinjury is a field of intensive research, especially for cryopreservation of organs. Vitrification using ethylene glycol and sucrose can be used in the freezing procedure to protect cartilage and this has been confirmed in vitro and in vivo [14,15]. However, protecting cartilage means protecting tumor cells in the epiphysis and it cannot be denied that vitrification increases the local recurrence rate.

There are only a few reports of clinical results of recycled osteoarticular autografts using methods such as irradiation and pasteurization. Koyanagi reported that 3 of 6 osteoarticular grafts after pasteurization failed because of late-onset absorption or infection with an average follow-up period of 165 months [16]. Poffyn reported that progressive joint narrowing occurred in all 26 osteoarticular reconstructions after irradiation with a mean follow-up of 135 months. The ISOLS radiological graft evaluation score was an average of $70.7 \%$ [17]. 
The use of allografts is more common clinically than the use of recycled autografts. Bus reviewed 514 cases of osteoarticular allografts and reported overall rates of failure of $27 \%$. They concluded that osteoarticular allograft reconstructions are associated with high rates of mechanical complications and did not justify routine employment of osteoarticular allografts for reconstruction of large joints after tumor resection [18]. Partial joint resection was reported by Muscolo for 38 patients who underwent unicondylar osteoarticular allografts that resulted in survival of $85 \%$ at 10 years [19]. Our findings are consistent with this report in that partial joint freezing produces promising results for osteoarticular recycling autografts. The reasons include the following. First, the smaller area of dead cartilage leads to better long-term survival. However, this is not sufficient to explain the good clinical outcome. Second, the joint stability could be preserved by intact ligaments and anatomical restoration. Instability is one of the major causes of collapse or common osteoarthritis. Reducing sacrifice of soft tissue might be as important as bony reconstruction. Third, as for the knee joint, the mechanical axis also plays an important role to preserve a single condyle. If the mechanical axis is on the spared condyle, it is a better surgical indication, because weight bearing on the grafted condyle can be avoided. In all our cases of unicondylar resection, the mechanical axis was on the preserved condyle.

The orientation of the tumor and precise osteotomy are quite important in partial joint resection for acetabular resection. We apply a navigation station and registration points registered within an error of $<1$ $\mathrm{mm}$ with 3D imaging. Once we determine the osteotomy site, bones were cut using a chisel linked to navigation, a bone saw, and a thread-wire saw. Although determining navigation settings requires 30-60 min, doubts about determining osteotomy sites are dramatically reduced [20].

If there is a large osteolytic lesion, or the patient requires early rehabilitation because of advanced age or advanced stage of malignancy, in our institution we consider a megaprosthesis is a better surgical indication for epiphyseal tumor. If the tumor location is limited in the epiphysis, partial joint resection and osteoarticular frozen autograft can be applied. Other tumors in an epiphysis should be reconstructed using a frozen autograft and prosthesis composite.

\section{Conclusion}

Grafted cartilage of frozen osteoarticular autografts was worn out over time. Hemicondylar resection or intraepiphyseal resection preserving partial healthy cartilage resulted in excellent survival. This technique is somewhat demanding and requires careful planning of the surgery but might be a reliable alternative to megaprosthesis.

\section{Abbreviations}

M: male; F: female; DOD: died of disease

\section{Declarations}




\section{Ethics approval and consent to participate}

This study was approved by the Institutional Review Board of the Kanazawa University Graduate School of Medical Science Kanazawa, Japan. All procedures were performed as part of standard medical care. Because this study was retrospective design and the researchers anonymized all of the data, the need for informed consent was waived by the Institutional Review Board.

\section{Consent for publication}

Institutional Review Board of the Kanazawa University Graduate School of Medical Science Kanazawa approved that the need for informed consent was waived by notification with opt-out.

\section{Availability of data and materials}

To protect privacy and respect confidentiality, no raw data have been made available in any public repository. The original operation reports, intraoperative photographs, imaging studies, and outpatient clinic records are retained as per the normal procedure within medical records of our institution. The datasets used and/or analysed during the current study available from the corresponding author on reasonable request.

\section{Competing interests}

The authors declare that they have no competing interests.

\section{Funding}

Not applicable.

\section{Authors' contributions}

All listed authors substantially contributed to the following aspects of the manuscript: $\mathrm{KH}, \mathrm{NY}, \mathrm{AT}, \mathrm{SM}, \mathrm{KI}$, and $\mathrm{HT}$ participated in diagnosing and treating the patient and in acquisition of data. $\mathrm{KH}, \mathrm{TH}, \mathrm{KA}, \mathrm{YT}, \mathrm{HA}$, $\mathrm{YA}, \mathrm{HY}$ and $\mathrm{SM}$ collected the findings and drafted the manuscript. $\mathrm{KH}$, and $\mathrm{HT}$ revised the manuscript. All authors read and approved the final manuscript. Dr. Hiroyuki Tsuchiya guarantee the integrity of this work. 


\section{Acknowledgments}

This manuscript has been previously published as an abstract at the 32nd Annual Meeting of the European Muscloskeletal Oncology Society.

\section{References}

1. Hayashi K, Tsuchiya H, Yamamoto N, Takeuchi A, Tomita K. Functional outcome in patients with osteosarcoma around the knee joint treated by minimised surgery. Int Orthop. 2008;32:63-8.

2. Watanabe K, Tsuchiya H, Yamamoto N, Shirai T, Nishida H, Hayashi K, et al. Over 10-year follow-up of functional outcome in patients with bone tumors reconstructed using distraction osteogenesis. $\mathrm{J}$ Orthop Sci. 2013;18:101-9.

3. Jamshidi K, Bahrabadi M, Mirzaei A, Tumors FB. Long-term Results of Osteoarticular Allograft Reconstruction in Children with Distal F emoral Bone Tumors. Arch bone Jt Surg. 2017;296 May 2016:296-301.

4. Tsuchiya H, Wan SL, Sakayama K, Yamamoto N, Nishida H, Tomita K. Reconstruction using an autograft containing tumour treated by liquid nitrogen. J Bone Joint Surg Br. 2005;87:218-25.

5. Shimozaki S, Yamamoto N, Shirai T, Nishida H, Hayashi K, Tanzawa Y, et al. Pedicle versus free frozen autograft for reconstruction in malignant bone and soft tissue tumors of the lower extremities. J Orthop Sci. 2014;19:156-63.

6. Takata M, Sugimoto N, Yamamoto N, Shirai T, Hayashi K, Nishida H, et al. Activity of bone morphogenetic protein-7 after treatment at various temperatures: Freezing vs. pasteurization vs. allograft. Cryobiology. 2011;63:235-9.

7. Nishida H, Shirai T, Hayashi K, Takeuchi A, Tanzawa Y, Mizokami A, et al. Cryotreatment against metastatic renal cell bone tumour reduced multiple lung metastases. Anticancer research. 2011;31:2927-30.

8. Tsuchiya H, Nishida H, Srisawat P, Shirai T, Hayashi K, Takeuchi A, et al. Pedicle frozen autograft reconstruction in malignant bone tumors. J Orthop Sci. 2010;15:340-9.

9. Igarashi K, Yamamoto N, Shirai T, Hayashi K, Nishida H, Kimura H, et al. The long-term outcome following the use of frozen autograft treated with liquid nitrogen in the management of bone and soft-tissue sarcomas. Bone Jt J. 2014;96 B:555-61.

10. Subhadrabandhu S, Takeuchi A, Yamamoto N, Shirai T, Nishida H, Hayashi K, et al. Frozen AutograftProsthesis Composite Reconstruction in Malignant Bone Tumors. Orthopedics. 2015;38:e911-8.

11. Chen W-M, Wu P-K, Chen C-F, Chung L-H, Liu C-L, Chen T-H. High-grade osteosarcoma treated with hemicortical resection and biological reconstruction. J Surg Oncol. 2012;105:825-9.

12. Miwa S, Taki J, Yamamoto N, Shirai T, Nishida H, Hayashi K, et al. A novel combined radiological method for evaluation of the response to chemotherapy for primary bone sarcoma. J Surg Oncol. 
2012;106:273-9.

13. Kanda Y. Investigation of the freely available easy-to-use software "EZR" for medical statistics. Bone Marrow Transplant. 2013;48:452-8.

14. Hayashi M, Tsuchiya H, Otoi T, Agung B, Yamamoto N, Tomita K. Cryobiology Influence of freezing with liquid nitrogen on whole-knee joint grafts and protection of cartilage from cryoinjury in rabbits. Cryobiology. 2009;59:28-35.

15. Onari I, Hayashi M, Ozaki N, Tsuchiya H. Cryobiology Vitreous preservation of articular cartilage from cryoinjury in rabbits. Cryobiology. 2012;65:98-103.

16. Koyanagi H, Matsumoto S, Shimoji T, Tanizawa T, Ae K, Shinomiya K, et al. Long-term results from use of pasteurized bone. J Orthop Sci. 2012;17:605-13.

17. Poffyn B, Sys G, Maele G Van, Verstraete K, Uyttendaele D, Van Maele G, et al. Radiographic analysis of extracorporeally irradiated autografts. Skeletal Radiol. 2010;39:999-1008.

18. Bus MPA, van de Sande MAJ, Taminiau AHM, Dijkstra PDS, Dijkstra S. Is there still a role for osteoarticular allograft reconstruction in musculoskeletal tumour surgery? Bone Joint J. 2017;99-B April:522-30.

19. Muscolo DL, Ayerza MA, Aponte-Tinao LA, Abalo E, Farfalli G. Unicondylar Osteoarticular Allografts of the Knee. J Bone Jt Surg. 2007;89:2137.

20. Abe K, Yamamoto N, Hayashi K, Takeuchi A, Miwa S, Igarashi K, et al. The usefulness of wide excision assisted by a computer navigation system and reconstruction using a frozen bone autograft for malignant acetabular bone tumors: a report of two cases. BMC Cancer. 2018;18:1036.

\section{Tables}

Due to technical limitations the Tables are only available as a download in the Supplementary Files.

Table 1. Characteristics of 27 patients who underwent osteoarticular grafting after freezing of tumorbearing bone

M: male, F: female, DOD: died of disease

\section{Additional Files}

File name: fig supp

Title of data: Number of cases for epiphyseal reconstruction using frozen autograft 
Description: Since 2003, 44 cases were reconstructed using a composite graft of frozen bone and a prosthesis instead of osteoarticular grafts.

\section{Figures}

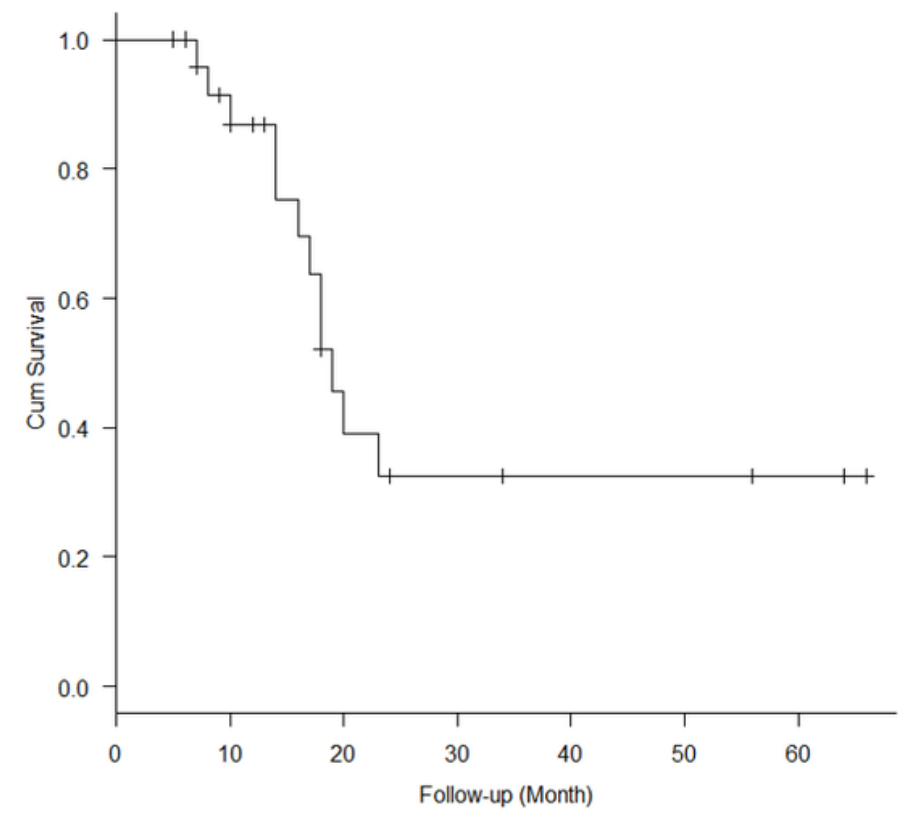

Fig. 1a

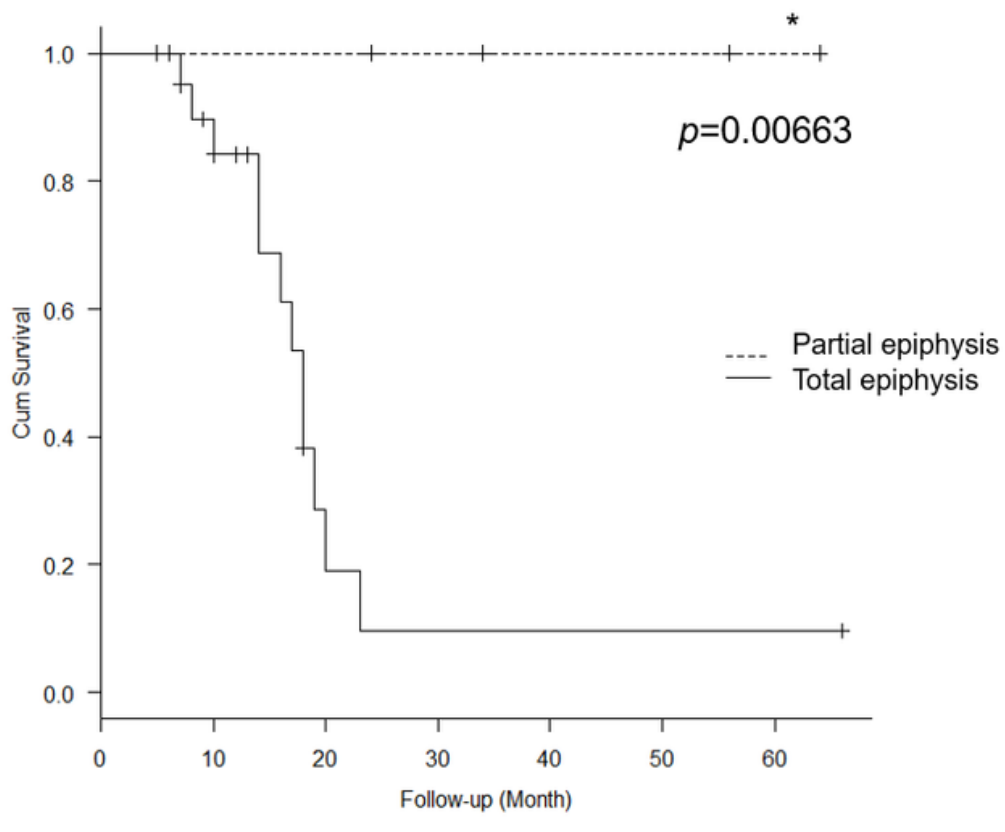

Fig. 1b

\section{Figure 1}

Survival of grafted cartilage The survival of grafted cartilage was assessed by X-ray imaging and the end point is defined as grade IV of the Kellgren and Lawrence osteoarthritis grading system. Survival of autografts was recorded using the Kaplan-Meier method and a log-rank test was used to determine significant difference between groups of patients with partial epiphyseal freezing and total epiphyseal freezing respectively. Patients who had died, who had deep infection, or who were lost to follow-up were censored for survival. a) 5-year survival rate of grafted frozen cartilage was $33 \%$. b) All patients in the group with partial epiphyseal freezing had survived at the final follow-up compared with only 1 patient who survived in the group with total epiphyseal freezing $(p=0.00663)$. 


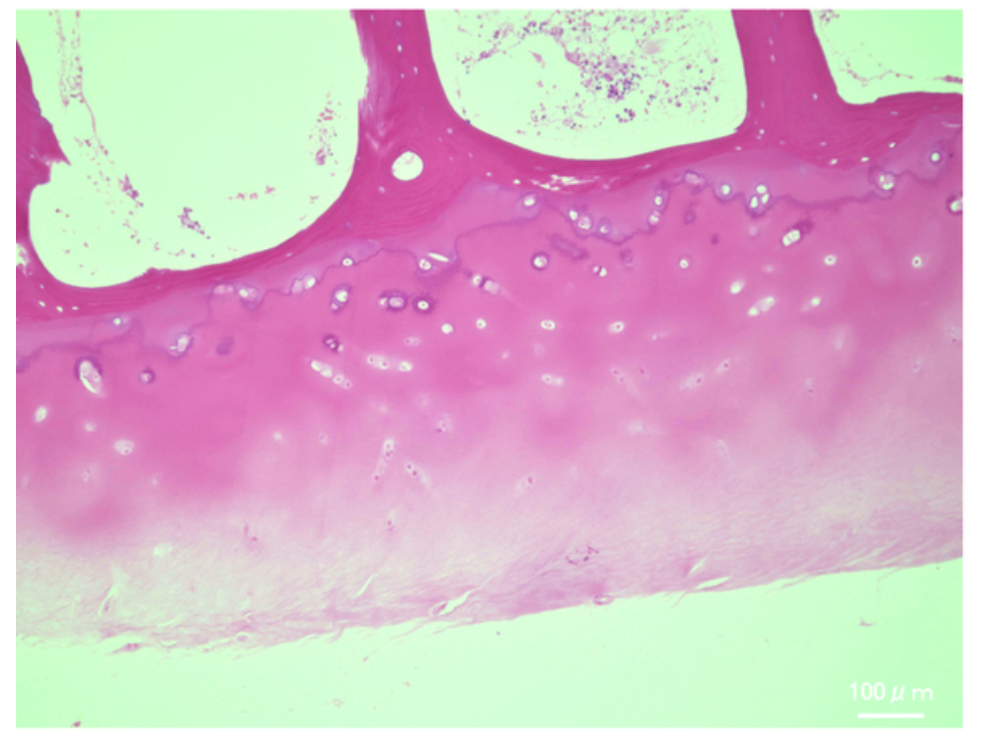

Fig. $2 a$

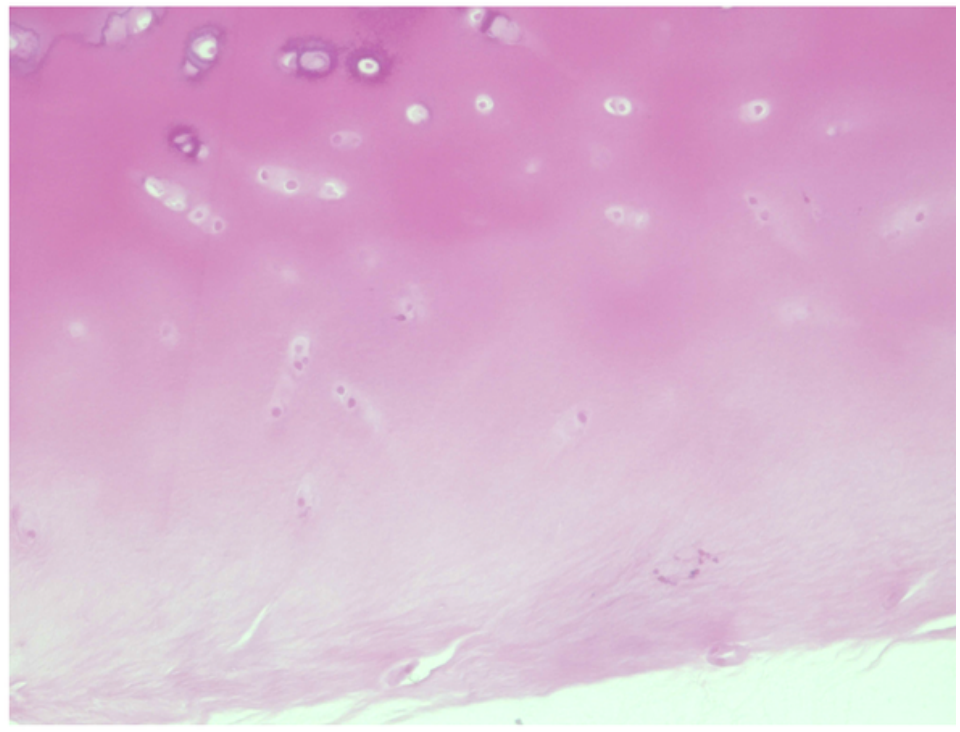

Fig. 2b

\section{Figure 2}

Case 7. The entire knee joint was resected and reconstructed with a frozen autograft. Because of infection, the grafted bone was excised 6 months later. The resected specimen with grafted cartilage was examined histologically. Hematoxylin and eosin staining showing dead cartilage with empty lacunae and the cartilage had surface fibrillation.

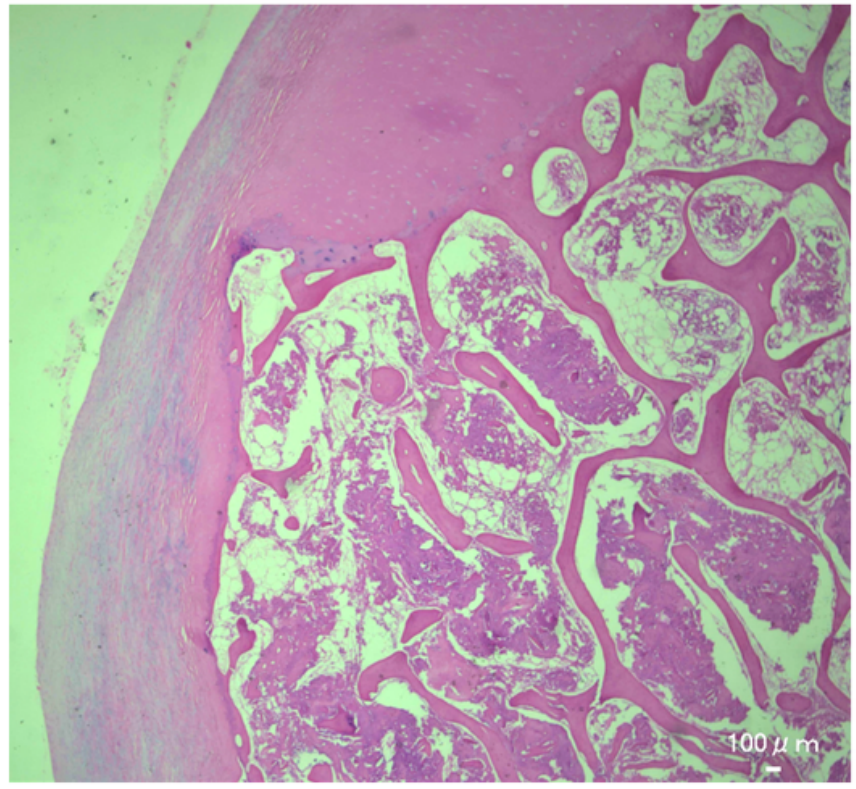

Fig. 3a

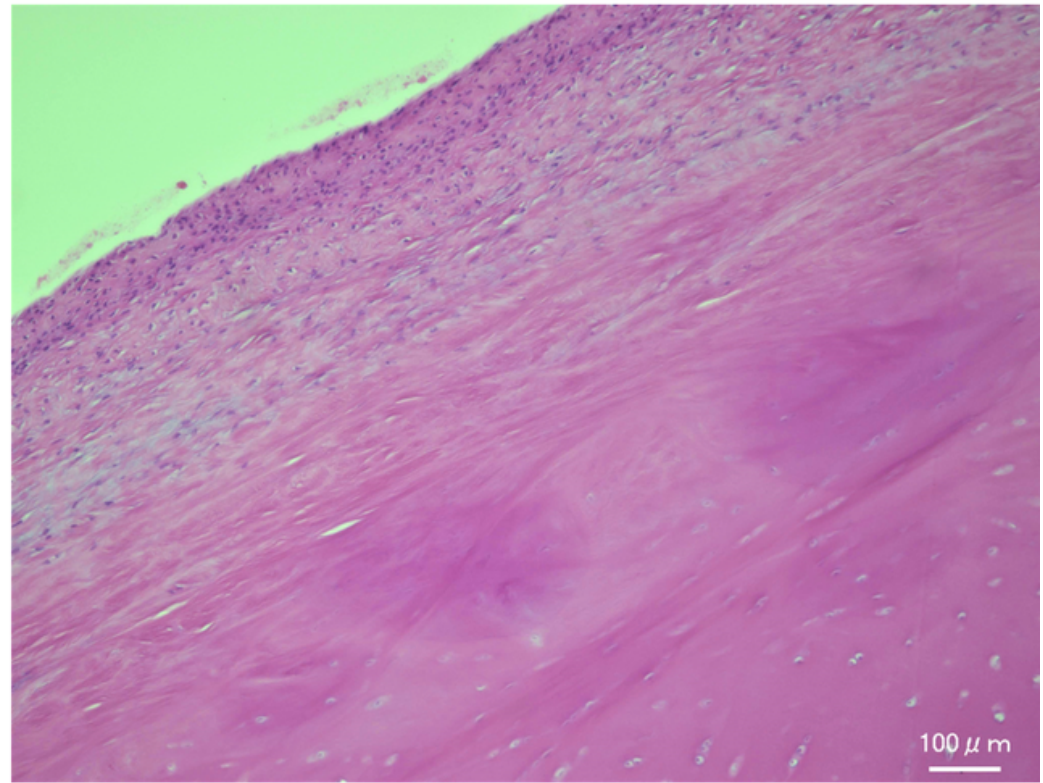

Fig. $3 b$ 
Figure 3

Case 5. Grafted frozen autograft of the distal femur was excised after 14 months because of collapse of the medial condyle. Histology showing dead cartilage with empty lacunae and the surface of the cartilage had reactive fibrous tissue.

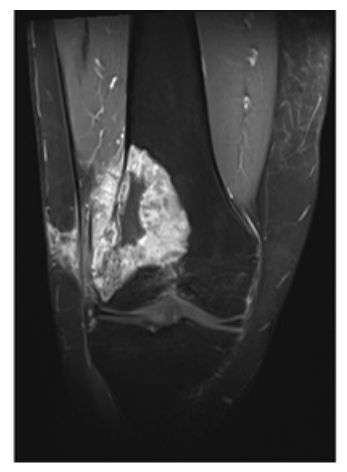

Fig. $4 a$

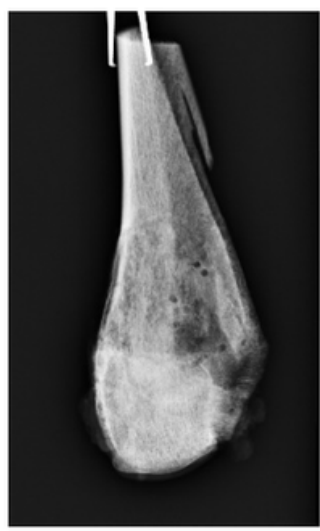

Fig. $4 b$

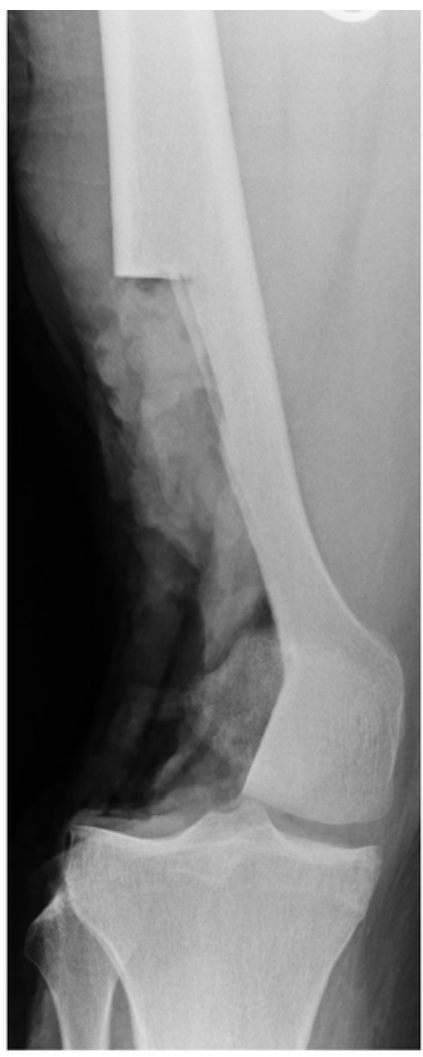

Fig. 4c

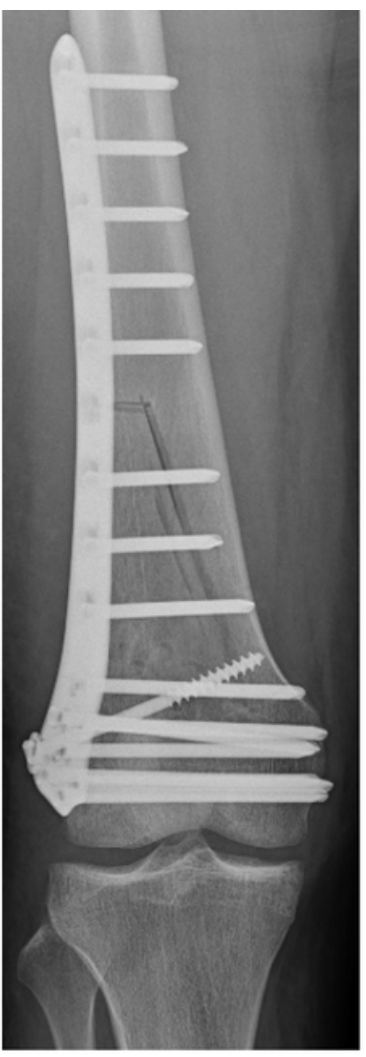

Fig. 4d

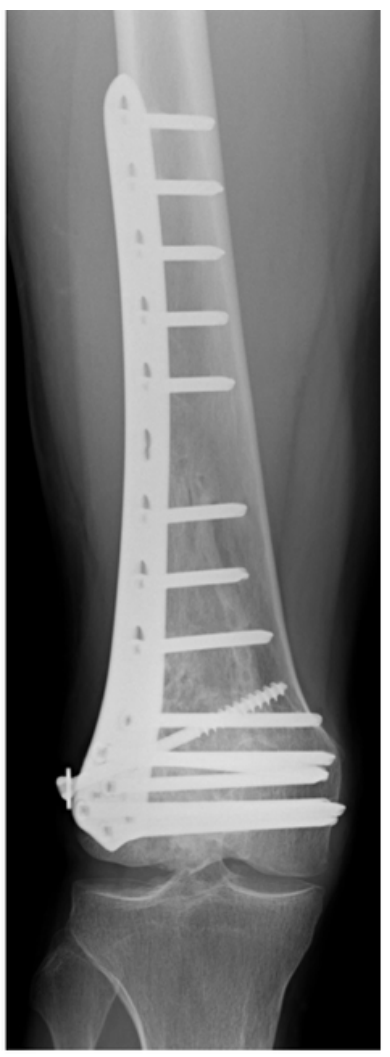

Fig. $4 \mathrm{e}$

\section{Figure 4}

Preservation of cartilage after partial resection of an epiphysis (case 25). a) A case of osteosarcoma of femoral lateral condyle with sufficient efficacy of preoperative chemotherapy. Only the lateral condyle was resected (b) and the medial condyle was preserved (c). d) The tumor-bearing bone was frozen in liquid nitrogen and reconstructed with plate. e) The joint is well preserved 56 months after the surgery. 


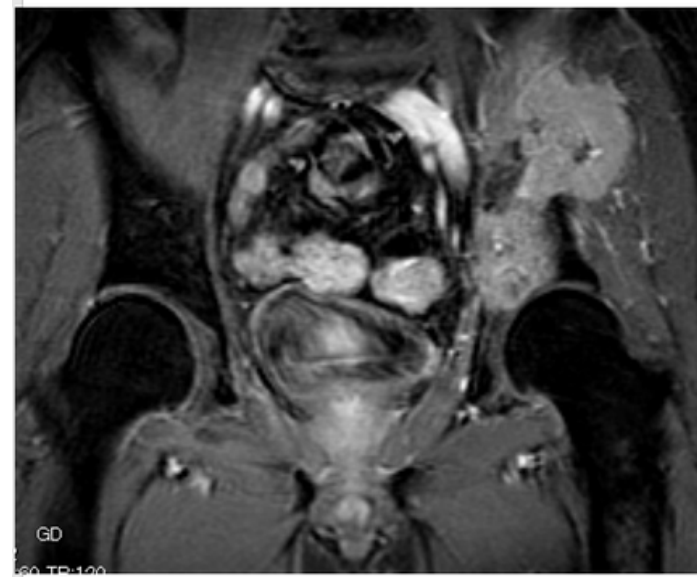

Fig. $5 a$

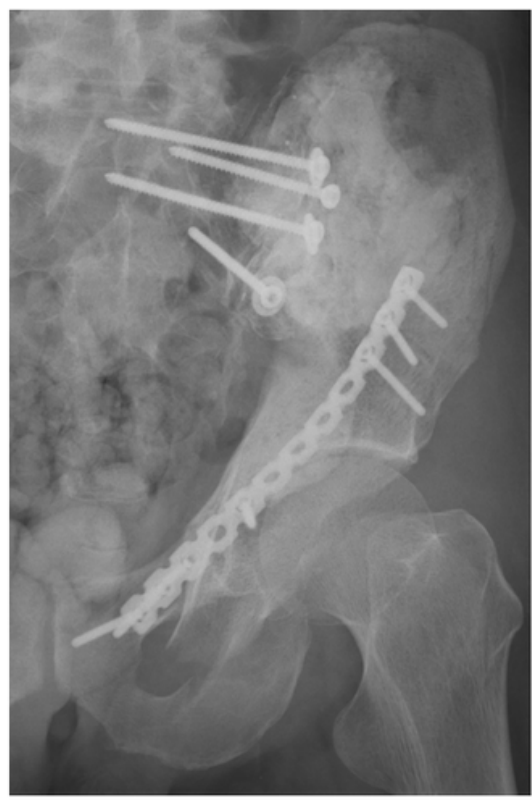

Fig. $5 b$

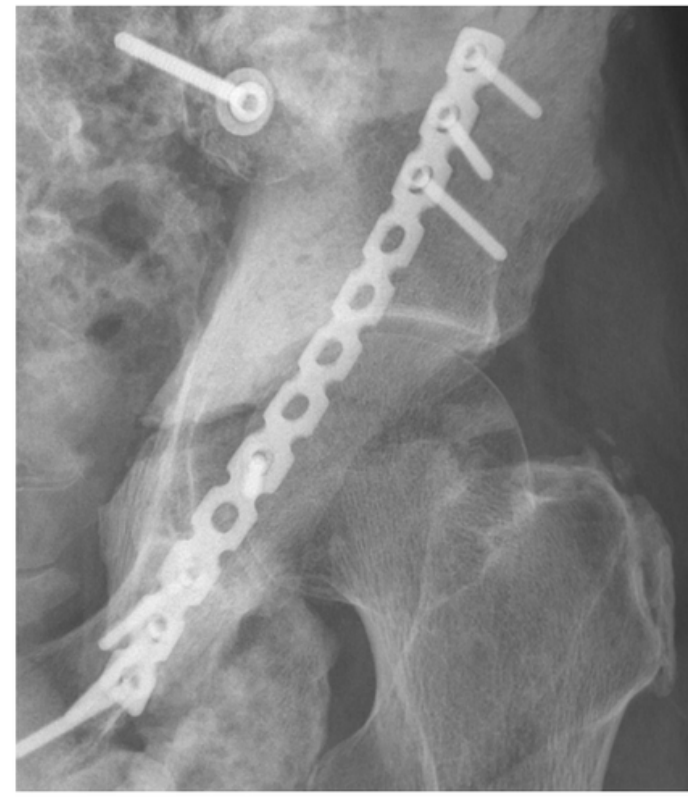

Fig. 5c

\section{Figure 5}

Preservation of cartilage after total resection of an epiphysis (case 11). a) A patient with metastatic tumor of the pelvis involving the acetabulum from a renal cell carcinoma. The entire acetabulum was resected and recycled after freezing. b) The osteolytic medial part filled with bone cement. c) There is no joint space narrowing 66 months after surgery.
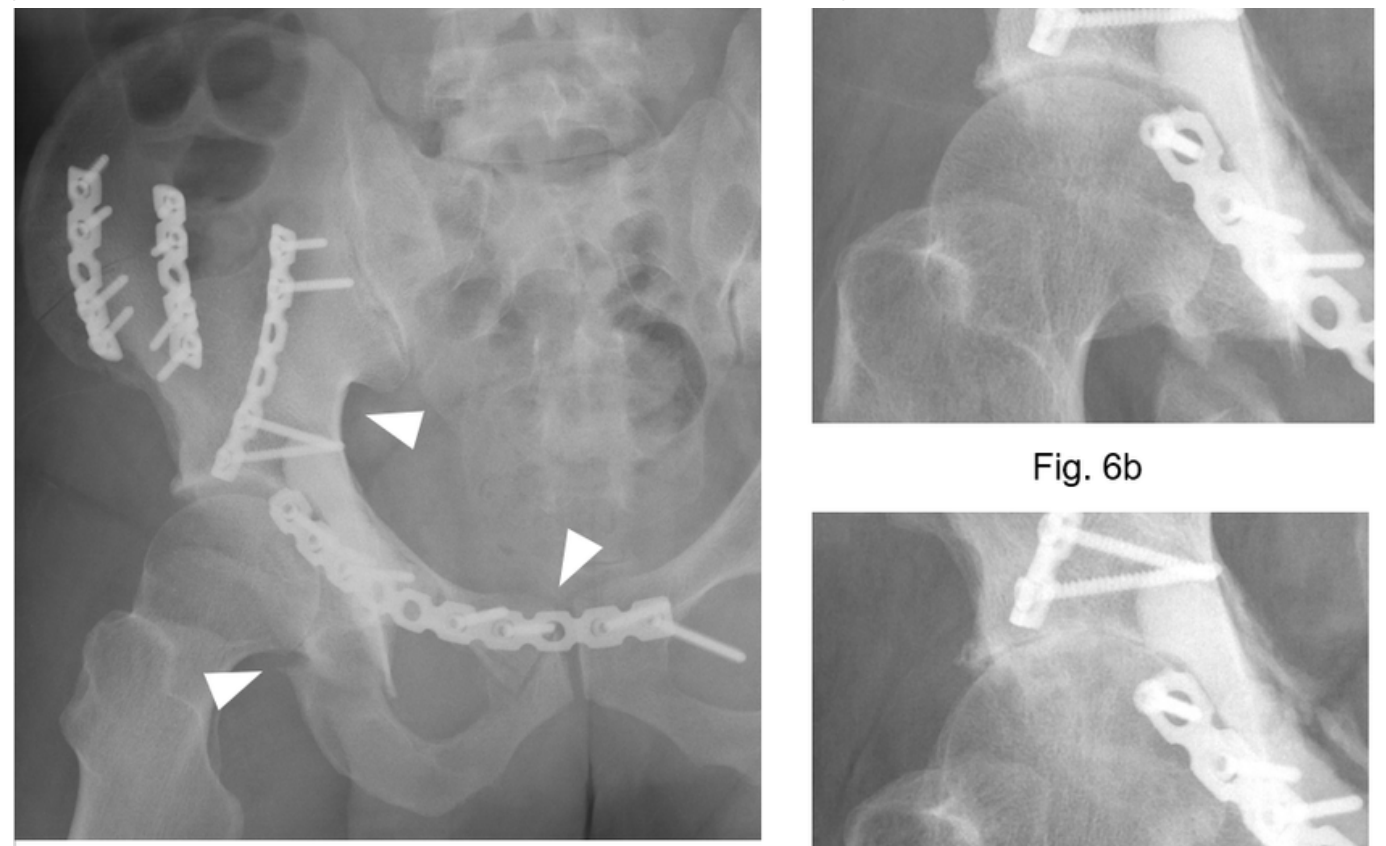

Fig. 6b

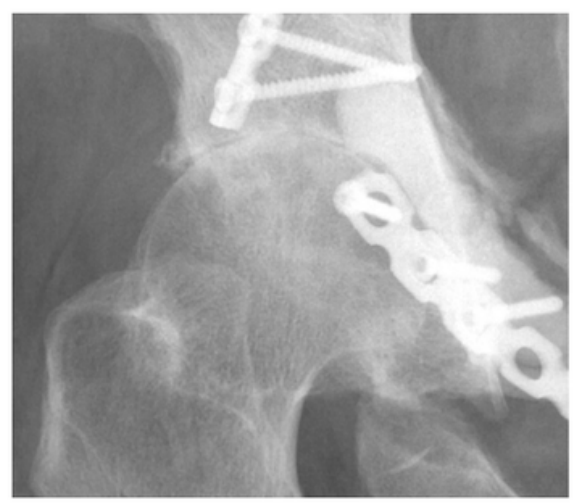

Fig. 6c

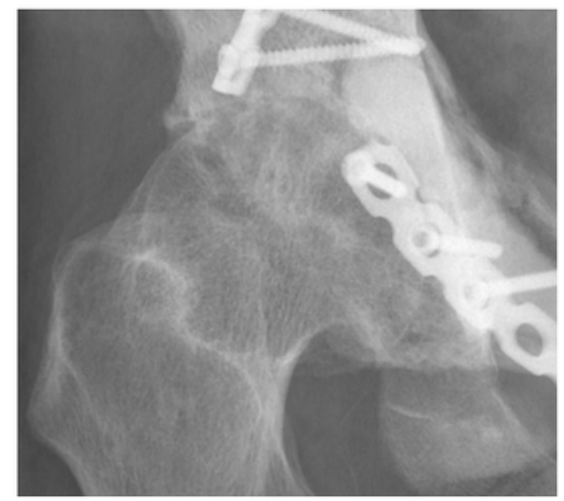

Fig. 6d 
Figure 6

Worn-out cartilage after total resection of an epiphysis (case 13). a) A patient with osteosarcoma of the pelvis after freezing of the acetabulum. Arrows show the region of the osteotomy. Sequential X-ray imaging reveals joint space narrowing over time (b, 13 months; $c, 23$ months; $d, 40$ months).

\section{Supplementary Files}

This is a list of supplementary files associated with this preprint. Click to download.

- table12.xlsx

- SupplementaryFigure.jpg 